\title{
M-convex Function on Generalized Polymatroid
}

\author{
Kazuo MUROTA* and Akiyoshi SHIOURA ${ }^{\dagger}$
}

April, 1997

\begin{abstract}
The concept of M-convex function, introduced recently by Murota, is a quantitative generalization of the set of integral points in an integral base polyhedron as well as an extension of valuated matroid of Dress-Wenzel (1990). In this paper, we extend this concept to functions on generalized polymatroids with a view to providing a unified framework for efficiently solvable nonlinear discrete optimization problems. The restriction of a function to $\left\{x \in \mathbf{Z}^{V} \mid x(V)=k\right\}$ for $k \in \mathbf{Z}$ is called a layer. We prove the M-convexity of each layer, and reveal that the minimizers in consecutive layers are closely related. Exploiting these properties, we can solve the optimization on layers efficiently. A number of equivalent exchange axioms are given for M-convex function on generalized polymatroid.
\end{abstract}

Keywords: matroid, base polyhedron, convex function, generalized polymatroid.

\section{Introduction}

In the area of discrete optimization, nonlinear optimization problems have been discussed as well as linear optimization problems. It is widely accepted that the well-solvability of linear optimization problems is deeply connected with polymatroid structures. In contrast, however, the essence of the well-solvability of nonlinear optimization problems has not been grasped clearly, while there are a number of scattered examples of efficiently solved nonlinear optimization problems such as the minimum convex-cost flow problem, the nonlinear resource allocation problem.

\footnotetext{
*Research Institute for Mathematical Sciences, Kyoto University, Kyoto 606-01, Japan, murota@kurims.kyoto-u.ac.jp.

${ }^{\dagger}$ Department of Mechanical Engineering, Sophia University, 7-1 Kioi-cho, Chiyoda-ku, Tokyo 102, Japan, shioura@keka.me.sophia.ac.jp. This work was done while the second author was at Research Institute for Mathematical Sciences, Kyoto University, as a short-term research fellow.
} 
On the other hand, discrete analogy of convex function has been considered by many authors, e.g., Miller [14], Lovász [13], Camerini et al. [1], and Favati-Tardella [7]. In particular, Lovász clarified the relationship between submodularity and convexity; Camerini et al. proposed the concept of quasi-separable convex function, showing the validity of a simple greedy algorithm for such functions; Favati-Tardella investigated a class of discrete functions such that local minimality leads to global minimality.

Recently, the concept of M-convex function has been introduced [19, 20, 21] as a quantitative generalization of the set of integral points in an integral base polyhedron. The concept of M-convex function provides us with a unified framework for efficiently solvable nonlinear optimization problems. A function $f: \mathbf{Z}^{V} \rightarrow \mathbf{R} \cup\{+\infty\}$ is said to be M-convex if it satisfies

(MB-EXC) $\forall x, y \in \operatorname{dom} f, \forall u \in \operatorname{supp}^{+}(x-y), \exists v \in \operatorname{supp}^{-}(x-y)$ such that

$$
f(x)+f(y) \geq f\left(x-\chi_{u}+\chi_{v}\right)+f\left(y+\chi_{u}-\chi_{v}\right)
$$

where $\operatorname{dom} f=\left\{x \in \mathbf{Z}^{V} \mid f(x)<+\infty\right\}, \operatorname{supp}^{+}(x-y)=\{w \in V \mid x(w)>y(w)\}$, $\operatorname{supp}^{-}(x-y)=\{w \in V \mid x(w)<y(w)\}$, and $\chi_{w} \in \mathbf{Z}^{V}$ is the characteristic vector of $w \in V$. The concept of M-convex function is also an extension of valuated matroid due to Dress and Wenzel $[5,6]$; for an M-convex function $f$ with $\operatorname{dom} f \subseteq\{0,1\}^{V},-f$ is a valuated matroid in the sense of $[5,6]$. The property (MB-EXC) implies that $\operatorname{dom} f$ is (the set of integral points of) a base polyhedron.

M-convex functions enjoy several nice properties: they can be extended to ordinary convex functions, and a Fenchel-type duality and a (discrete) separation theorem hold for them $[16,19,20,21]$. These properties may be sufficient for us to regard M-convexity as convexity in discrete optimization. Applications of M-convex functions for polynomial matrices are described in $[5,6,15]$.

The main aim of this paper is to extend the concept of M-convexity to functions on generalized polymatroids. The concept of generalized polymatroid, or g-polymatroid for short, was introduced by Frank [8] in 1981 (see also Tardos [24] and Frank and Tardos [9]). G-polymatroid includes polymatroid, submodular polyhedron, supermodular polyhedron, and base polyhedron as its special cases. Although g-polymatroid is a generalization of those polyhedra mentioned above, it is also known to be equivalent to base polyhedron in the sense that any g-polymatroid can be obtained as a projection of a base polyhedron. Given a set $Q\left(\subseteq \mathbf{Z}^{V}\right)$, define $\widetilde{Q}\left(\subseteq \mathbf{Z}^{V \cup\left\{v_{0}\right\}}\right)$ as $\widetilde{Q}=\left\{(x,-x(V)) \in \mathbf{Z}^{V \cup\left\{v_{0}\right\}} \mid x \in Q\right\}$, where $v_{0}$ is a new element not in $V$, and $x(V)=\sum\{x(w) \mid w \in V\}$.

Theorem 1.1 (Fujishige $[\mathbf{1 0}, \mathbf{1 1}]) Q$ is (the set of integral points in) a g-polymatroid if and only if

(G-PRJ) $\widetilde{Q}$ is (the set of integral points in) a base polyhedron. 
In view of this theorem, it would be natural to define M-convexity for a function on a gpolymatroid as follows: a function $f: \mathbf{Z}^{V} \rightarrow \mathbf{R} \cup\{+\infty\}$ is defined to be M-convex on a g-polymatroid if

(MG-PRJ) function $\tilde{f}: \mathbf{Z}^{V \cup\left\{v_{0}\right\}} \rightarrow \mathbf{R} \cup\{+\infty\}$ satisfies (MB-EXC), where

$$
\widetilde{f}\left(x, x_{0}\right)= \begin{cases}f(x) & \left(x_{0}=-x(V)\right), \\ +\infty & \text { (otherwise) }\end{cases}
$$

It is clear that $\operatorname{dom} f$ is indeed a g-polymatroid for any M-convex function $f$ on a gpolymatroid.

Though M-convexity on a g-polymatroid is a straightforward translation of M-convexity on a base polyhedron, we believe that it is worth investigating in its own right. One motivation is that we can talk of the layer structure of an M-convex function when it is defined on a g-polymatroid, where a layer of a function is defined as its restriction to $\left\{x \in \mathbf{Z}^{V} \mid x(V)=k\right\}$ for each $k \in \mathbf{Z}$. Then optimization on each layer naturally comes into a problem. Recently, many researchers analyze set systems and functions with respect to layer structures; for example, greedoid by Korte, Lovász, and Schrader [12], valuated bimatroid [15], valuation on independent sets [17], well-layered map and rewarding map by Dress and Terhalle $[2,3,4]$, and so on. In particular, valuations on independent sets enjoy M-concavity on g-polymatroids, i.e., the negative of M-convex functions. We show that optimization of an M-convex function in a specified layer can be done efficiently in several different ways.

Another motivation is the richness of examples of M-convex functions on g-polymatroids, e.g., network flows, location problems, and polynomial matrices (see Section 2). It is well known that kinds of greedy algorithms work for those problems, but such phenomena cannot be explained by the theory of g-polymatroid. The framework of M-convex functions on gpolymatroids explains why greedy algorithms work well for those problems.

In view of the exchange axiom (MB-EXC) for an M-convex function on a base polyhedron, it would be natural to ask how the M-convexity on a g-polymatroid, defined through projection, can be characterized by inherent exchange properties. We show in Theorem 4.2 that an M-convex function on a g-polymatroid is characterized by either of the following simultaneous exchange properties:

(MG-EXC) $\forall x, y \in \operatorname{dom} f, \forall u \in \operatorname{supp}^{+}(x-y)$, $f(x)+f(y) \geq \min \left[f\left(x-\chi_{u}\right)+f\left(y+\chi_{u}\right), \min _{v \in \operatorname{supp}^{-}(x-y)}\left\{f\left(x-\chi_{u}+\chi_{v}\right)+f\left(y+\chi_{u}-\chi_{v}\right)\right\}\right]$,

$(\mathbf{M G}-\mathbf{E X C} \mathbf{W}) \forall x, y \in \operatorname{dom} f$ with $x(V) \geq y(V)$ and $x \neq y$,

$f(x)+f(y) \underset{u \in \operatorname{supp}^{+}(x-y)}{\geq}\left[f\left(x-\chi_{u}\right)+f\left(y+\chi_{u}\right), \min _{v \in \operatorname{supp}^{-}(x-y)}\left\{f\left(x-\chi_{u}+\chi_{v}\right)+f\left(y+\chi_{u}-\chi_{v}\right)\right\}\right]$. 
This paper is organized as follows: Section 2 provides various examples of M-convex functions on g-polymatroids. Section 3 discusses the layer structure of M-convex function and minimization on layers. Finally, we show the equivalence of a number of exchange axioms in Section 4.

\section{Examples of M-convex Functions on G-polymatroids}

M-convex functions on g-polymatroids arise naturally in discrete optimization as follows. While Examples 2.2 and 2.4 are minor variants of known facts, the others are new observations.

Example 2.1 (Quasi-separable convex function) Let $f: \mathbf{Z}^{V} \rightarrow \mathbf{R} \cup\{+\infty\}$. For any $x \in \operatorname{dom} f$ and $u \in V$ with $x+\chi_{u} \in \operatorname{dom} f$, we define a discrete derivative of $f$ in direction $u$ at $x$ as $\partial_{u} f(x)=f\left(x+\chi_{u}\right)-f(x)$. Camerini et al. [1] called $f$ quasi-separable convex if there exist functions $\Phi_{u}(u \in V)$ such that for each $u \in V, \Phi_{u}$ satisfies $\partial_{u} f(x)=\Phi_{u}(x(V), x(u))$ and $\Phi_{u}$ is nondecreasing w.r.t. both $x(V)$ and $x(u)$. For example, a quadratic function

$$
f(x)=\sum_{i=1}^{n} a_{i} x_{i}^{2}+b \sum_{i<j} x_{i} x_{j}
$$

is quasi-separable convex when $0 \leq b \leq 2 \min \left\{a_{i} \mid 1 \leq i \leq n\right\}$. We can show that a quasiseparable convex function $f$ is M-convex on a g-polymatroid when $\operatorname{dom} f$ is a polymatroid.

Example 2.2 (Min-cost flow) Let $G=\left(V, A ; V^{+}, V^{-}\right)$be a directed graph with two specified vertex sets $V^{+}, V^{-} \subseteq V$ such that $V^{+} \cap V^{-}=\emptyset$. We denote an upper capacity function by $\bar{c}: A \rightarrow \mathbf{Z} \cup\{+\infty\}$, a lower capacity function by $\underline{c}: A \rightarrow \mathbf{Z} \cup\{-\infty\}$. A flow is a function $\varphi: A \rightarrow \mathbf{Z}$, and its boundary $\partial \varphi: V \rightarrow \mathbf{Z}$ is defined as

$$
\partial \varphi(v)=\sum\{\varphi(a) \mid a \text { leaves } v\}-\sum\{\varphi(a) \mid a \text { enters } v\} \quad(v \in V) .
$$

A flow $\varphi$ is called feasible if it satisfies $\underline{c}(a) \leq \varphi(a) \leq \bar{c}(a)(\forall a \in A)$ and $\partial \varphi(v)=0(\forall v \in$ $\left.V-\left(V^{+} \cup V^{-}\right)\right)$. Then, we see that $Q=\left\{(\partial \varphi)^{-} \mid \varphi\right.$ : feasible flow $\}\left(\subseteq \mathbf{Z}^{V^{-}}\right)$is a g-polymatroid, where $(\partial \varphi)^{-}$is the restriction of $\partial \varphi$ to $V^{-}$.

Suppose we are given a family of convex functions $f_{a}: \mathbf{Z} \rightarrow \mathbf{R}$ indexed by $a \in A$. Here we call $f_{a}$ convex if its piecewise linear extension $\bar{f}_{a}: \mathbf{R} \rightarrow \mathbf{R}$ is an ordinary convex function. We define a function $f_{\text {mcf }}: \mathbf{Z}^{V^{-}} \rightarrow \mathbf{R} \cup\{ \pm \infty\}$ as follows:

$$
f_{\mathrm{mcf}}(x)= \begin{cases}\inf \left\{\Gamma(\varphi) \mid \varphi: \text { feasible flow, }(\partial \varphi)^{-}=x\right\} & (x \in Q), \\ +\infty & (x \notin Q),\end{cases}
$$

where $\Gamma(\varphi)=\sum\left\{f_{a}(\varphi(a)) \mid a \in A\right\}$. Then, the function $f_{\text {mcf }}$ satisfies (MG-EXC) if $f_{\text {mcf }}$ does not take the value $-\infty$, which can be proved in the similar way as in [19, 21]. 
Example 2.3 ( $\boldsymbol{k}$-tree-core) Suppose we are given a tree network $T=(V, E)$ with an edge length function $l: E \rightarrow \mathbf{R}_{+}$and a vertex weight function $w: V \rightarrow \mathbf{R}_{+}$. For any $u, v \in V$, denote by $P(u, v)$ the unique path connecting $u$ and $v$. We define the distance $d(u, v)$ between $u, v \in V$ as the sum of lengths of edges in $P(u, v)$. The distance-sum $\operatorname{dis}(S)$ of a subtree (connected subgraph) $S$ is given by

$$
\operatorname{dis}(S)=\sum_{u \in V} w(u) \cdot \min _{v \in S} d(u, v) .
$$

A $k$-tree-core is a subtree with $k$ leaves minimizing the distance-sum. We represent each subtree of $T$ by the set of its leaves. Put

$$
\mathcal{F}=\{X \subseteq V|| X \mid \geq 2, X \text { is the leaf set of some subtree }\}
$$

and denote by $S(X)$ the subtree corresponding to $X \in \mathcal{F}$. Define a function $f_{\text {dis }}: \mathbf{Z}^{V} \rightarrow$ $\mathbf{R} \cup\{+\infty\}$ by

$$
f_{\text {dis }}(x)= \begin{cases}\operatorname{dis}(S(X)) & \left(x=\chi_{X} \text { for some } X \in \mathcal{F}\right), \\ +\infty & \text { (otherwise), }\end{cases}
$$

where $\chi_{X} \in\{0,1\}^{V}$ is the characteristic vector of $X \subseteq V$. Then, $f_{\text {dis }}$ satisfies (MG-EXC). See Peng et al.[22] and Shioura and Uno [23] for more about $k$-tree-core.

Example 2.4 (Polynomial matrices $[\mathbf{3}, \mathbf{5}, \mathbf{6}, \mathbf{1 5}]$ ) Let $A(t)$ be an $m \times n$ polynomial matrix, where each entry of $A(t)$ is a polynomial in $t$. Denote by $R$ and $C$ the row and column sets of $A(t)$, respectively. Define $\mathcal{J}$ to be the family of linearly independent column sets, and $f_{\text {mat }}: \mathbf{Z}^{C} \rightarrow \mathbf{R} \cup\{+\infty\}$ by

$$
f_{\text {mat }}(x)= \begin{cases}-\max \left\{\operatorname{deg}_{t} \operatorname{det} A[I, J]|I \subseteq R,| I|=| J \mid\right\} & \left(x=\chi_{J}, J \in \mathcal{J}\right), \\ +\infty & \text { (otherwise) }\end{cases}
$$

where $A[I, J]$ is the submatrix of $A(t)$ induced by the row set $I$ and the column set $J$. Then, we can show that the function $f_{\text {mat }}$ satisfies (MG-EXC) by using the Grassmann-Plücker identity.

\section{$3 \quad$ Greedily Solvable Layer Structure}

Suppose we are given a function $f: \mathbf{Z}^{V} \rightarrow \mathbf{R} \cup\{+\infty\}$. This section assumes that $f$ satisfies (MG-EXC), i.e., $f$ is M-convex on a g-polymatroid, unless otherwise stated explicitly. We discuss the layer structure of $f$, which is the restriction of $f$ to $\left\{x \in \mathbf{Z}^{V} \mid x(V)=k\right\}$, and the following optimization problem in each layer $(k \in \mathbf{Z})$ :

$$
\text { minimize } f(x) \text { subject to } x(V)=k \text {. }
$$

Set $\lambda=\min \{x(V) \mid f(x)<+\infty\}$ and $\mu=\max \{x(V) \mid f(x)<+\infty\}$. For any integer $k$, define a function $f_{k}: \mathbf{Z}^{V} \rightarrow \mathbf{R} \cup\{+\infty\}$ as $f_{k}(x)=f(x)$ if $x(V)=k$, and $=+\infty$ otherwise. The following shows that each layer has a nice structure. 
Theorem $3.1 f_{k}$ satisfies $(M B-E X C)(\lambda \leq \forall k \leq \mu)$.

Proof. This is an immediate corollary of Theorem 4.2 to be established in Section 4 .

We can find a minimizer in each layer greedily by the following algorithm.

\section{Exchanging Algorithm}

STEP 0: Let $x$ be any element in $\operatorname{dom} f$. Set $V^{-}=V$.

STEP 1: If $V^{-}=\emptyset$ then stop.

STEP 2: Choose any $u \in V^{-}$, and find $v \in V$ such that $f\left(x-\chi_{u}+\chi_{v}\right)=\min \left\{f\left(x-\chi_{u}+\chi_{w}\right) \mid\right.$ $w \in V\}$.

SteP 3: Set $x=x-\chi_{u}+\chi_{v}$, and if $v \in V^{-}$then set $V^{-}=V^{-}-\{v\}$. Go to SteP 1.

Note that with a slight modification, this algorithm also applies to global optimization for M-convex functions on g-polymatroids. The next lemma validates the exchanging algorithm.

Lemma 3.2 Suppose $f: \mathbf{Z}^{V} \rightarrow \mathbf{R} \cup\{+\infty\}$ satisfies (MB-EXC). Given $x \in \operatorname{dom} f$ and $u \in V$, let $v \in V$ be such that $f\left(x-\chi_{u}+\chi_{v}\right)=\min \left\{f\left(x-\chi_{u}+\chi_{w}\right) \mid w \in V\right\}$.

(i) If $v \neq u$, there exists $x^{*} \in \arg \min f$ with $x^{*}(v)>x(v)$.

(ii) If $v=u$, there exists $x^{*} \in \arg \min f$ with $x^{*}(v) \geq x(v)$.

Proof. We prove the first claim only. The second claim can be proved in a similar way. Let $x^{*} \in \arg \min f$ with the maximum value of $x^{*}(v)$, and to the contrary suppose $x^{*}(v) \leq x(v)$. Then we have $v \in \operatorname{supp}^{+}\left(\left(x-\chi_{u}+\chi_{v}\right)-x^{*}\right)$. By (MB-EXC), there exists $w \in \operatorname{supp}^{-}\left(\left(x-\chi_{u}+\chi_{v}\right)-x^{*}\right)$ such that

$$
f\left(x-\chi_{u}+\chi_{v}\right)+f\left(x^{*}\right) \geq f\left(x-\chi_{u}+\chi_{w}\right)+f\left(x^{*}+\chi_{v}-\chi_{w}\right) .
$$

The assumption for $v$ and the fact $x^{*} \in \arg \min f$ imply $f\left(x^{*}+\chi_{v}-\chi_{w}\right)=f\left(x^{*}\right)$. However, it is a contradiction since $\left(x^{*}+\chi_{v}-\chi_{w}\right)(v)=x^{*}(v)+1$.

We propose different approaches for optimization in a layer, which use the following properties on the relationship between consecutive layers. For any integer $k(\lambda \leq k \leq \mu)$, define $\alpha_{k}^{*}=\min \{f(x) \mid x(V)=k\}$ and $M_{k}=\left\{x \in \mathbf{Z}^{V} \mid x(V)=k, f(x)=\alpha_{k}^{*}\right\}$. For any $x \in \mathbf{Z}^{V}$, we define $\|x\|=\sum\{|x(w)| \mid w \in V\}$.

Theorem 3.3 (i) Let $x_{k}^{*} \in M_{k}(\lambda \leq k \leq \mu-1)$, and $v \in V$ be such that $f\left(x_{k}^{*}+\chi_{v}\right)=$ $\min \left\{f\left(x_{k}^{*}+\chi_{w}\right) \mid w \in V\right\}$. Then $x_{k}^{*}+\chi_{v} \in M_{k+1}$.

(ii) Let $x_{k}^{*} \in M_{k}(\lambda+1 \leq k \leq \mu)$ and $u \in V$ be such that $f\left(x_{k}^{*}-\chi_{u}\right)=\min \left\{f\left(x_{k}^{*}-\chi_{w}\right) \mid\right.$ $w \in V\}$. Then $x_{k}^{*}-\chi_{u} \in M_{k-1}$.

Proof. For (i) it suffices to show that $\left\|y^{*}-x_{k}^{*}\right\|=1$ holds for some $y^{*} \in M_{k+1}$. Let $y \in M_{k+1}$ with $\left\|y-x_{k}^{*}\right\|>1$. Note that $\operatorname{supp}^{+}\left(y-x_{k}^{*}\right) \neq \emptyset$. For $u \in \operatorname{supp}^{+}\left(y-x_{k}^{*}\right)$, the property (MG-EXC) yields either (a) or (b), where

(a) $f(y)+f\left(x_{k}^{*}\right) \geq f\left(y-\chi_{u}\right)+f\left(x_{k}^{*}+\chi_{u}\right)$, 
(b) $f(y)+f\left(x_{k}^{*}\right) \geq f\left(y-\chi_{u}+\chi_{v}\right)+f\left(x_{k}^{*}+\chi_{u}-\chi_{v}\right) \quad\left(\exists v \in \operatorname{supp}^{-}\left(y-x_{k}^{*}\right)\right)$.

Since $x_{k}^{*} \in M_{k}, y \in M_{k+1}$, we have $x_{k}^{*}+\chi_{u} \in M_{k+1}$ if (a) holds, and $y-\chi_{u}+\chi_{v} \in M_{k+1}$ if (b) holds. If (a) holds, we are done. In case of (b), we obtain $y^{\prime}=y-\chi_{u}+\chi_{v} \in M_{k+1}$ with $\left\|y^{\prime}-x_{k}^{*}\right\|<\left\|y-x_{k}^{*}\right\|$. By repeating this procedure, we can find a desired $y^{*}$. The proof of (ii) is similar.

This property naturally yields the next algorithm:

\section{Augmenting Algorithm}

SteP 0: Find any $x_{\lambda}^{*} \in M_{\lambda}$. Set $k=\lambda$.

STEP 1: If $k=\mu$ then stop.

STEP 2: Find $v_{k} \in V$ such that $f\left(x_{k}^{*}+\chi_{v_{k}}\right)=\min \left\{f\left(x_{k}^{*}+\chi_{w}\right) \mid w \in V\right\}$.

SteP 3: Set $x_{k+1}^{*}=x_{k}^{*}+\chi_{v_{k}}, k=k+1$. Go to SteP 1 .

The exchanging algorithm can be used in STEP 0 of this algorithm. A reducing algorithm, which iteratively reduces $k$, can be constructed similarly. These algorithms work well if we can find an element $x_{\lambda}^{*} \in M_{\lambda}$ or $x_{\mu}^{*} \in M_{\mu}$ efficiently, in particular if $\left|\operatorname{dom} f_{\lambda}\right|=1$ or $\left|\operatorname{dom} f_{\mu}\right|=1$.

The next theorem shows the convexity of the sequence $\alpha_{k}^{*}$.

Theorem $3.4 \alpha_{k-1}^{*}+\alpha_{k+1}^{*} \geq 2 \alpha_{k}^{*} \quad(\lambda+1 \leq \forall k \leq \mu-1)$.

Proof. By Theorem 3.3, there exist $x_{k-1}^{*} \in M_{k-1}, x_{k+1}^{*} \in M_{k+1}$ such that $x_{k-1}^{*} \leq x_{k+1}^{*}$. Apply (MG-EXC) to $x_{k+1}^{*}, x_{k-1}^{*}$ and any $u \in \operatorname{supp}^{+}\left(x_{k+1}^{*}-x_{k-1}^{*}\right)$ to obtain $f\left(x_{k+1}^{*}\right)+$ $f\left(x_{k-1}^{*}\right) \geq f\left(x_{k+1}^{*}-\chi_{u}\right)+f\left(x_{k-1}^{*}+\chi_{u}\right) \geq 2 \alpha_{k}^{*}$. Note that $\operatorname{supp}^{-}\left(x_{k+1}^{*}-x_{k-1}^{*}\right)=\emptyset$.

Therefore, we can use the augmenting algorithm for finding a global minimum, where we can stop the algorithm when $k$ satisfies the condition $\alpha_{k+1}^{*} \geq \alpha_{k}^{*}$. As an immediate corollary of this theorem, we have $\left\{x \in \mathbf{Z}^{V} \mid x(V)=k, f(x)<+\infty\right\} \neq \emptyset(\lambda \leq \forall k \leq \mu)$.

Finally, we mention that the local minimality characterizes a global minimum of an Mconvex function on a g-polymatroid. This follows easily from the corresponding result $[19,20]$ for an M-convex function on a base polyhedron.

Theorem 3.5 Let $x \in \operatorname{dom} f$. Then, $f(x) \leq f(y)$ for any $y \in \mathbf{Z}^{V}$ if and only if

$$
f(x) \leq \min \left[\min _{u, v \in V} f\left(x-\chi_{u}+\chi_{v}\right), \min _{u \in V} f\left(x-\chi_{u}\right), \min _{v \in V} f\left(x+\chi_{v}\right)\right] .
$$

Remark 3.1 The validity of the greedy algorithms in [1] is explained by the results in this section together with the M-convexity of quasi-separable convex functions. 


\section{Exchange Axioms for M-convex Functions on G-polymatroids}

We derive here a number of equivalent exchange axioms for M-convex functions on $\mathrm{g}$ polymatroids. ${ }^{1}$ We first recall a seemingly weaker exchange property than (MB-EXC) for M-convex functions on base polyhedra:

(MB-EXC $\mathbf{W}) \forall x, y \in \operatorname{dom} f$ with $x \neq y, \exists u \in \operatorname{supp}^{+}(x-y), \exists v \in \operatorname{supp}^{-}(x-y)$ such that $f(x)+f(y) \geq f\left(x-\chi_{u}+\chi_{v}\right)+f\left(y+\chi_{u}-\chi_{v}\right)$.

Theorem $4.1([\mathbf{1 8}, \mathbf{2 0}])(M B-E X C) \Longleftrightarrow\left(M B-E X C_{w}\right)$.

This equivalence is a quantitative generalization of the result of Tomizawa [25] for base polyhedra.

A straightforward translation of (MB-EXC) and $\left(\mathrm{MB}^{\left.-E X C_{\mathrm{W}}\right)}\right.$ through the equation (1) leads to the following exchange axioms for M-convex functions on g-polymatroids:

$(\mathbf{M G}-\mathbf{E X C} \mathbf{p}) \forall x, y \in \operatorname{dom} f$,

(i) $x(V)<y(V) \Longrightarrow f(x)+f(y) \underset{v \in \operatorname{supp}^{-}(x-y)}{\geq}\left\{f\left(x+\chi_{v}\right)+f\left(y-\chi_{v}\right)\right\}$,

(ii) $x(V) \leq y(V) \Longrightarrow \forall u \in \operatorname{supp}^{+}(x-y)$,

$$
f(x)+f(y) \underset{v \in \operatorname{supp}^{-}(x-y)}{\geq}\left\{f\left(x-\chi_{u}+\chi_{v}\right)+f\left(y+\chi_{u}-\chi_{v}\right)\right\},
$$

(iii) $x(V)>y(V) \Longrightarrow \forall u \in \operatorname{supp}^{+}(x-y)$,

$$
f(x)+f(y) \geq \min \left[f\left(x-\chi_{u}\right)+f\left(y+\chi_{u}\right), \min _{v \in \operatorname{supp}^{-}(x-y)}\left\{f\left(x-\chi_{u}+\chi_{v}\right)+f\left(y+\chi_{u}-\chi_{v}\right)\right\}\right],
$$

(MG-EXC $\mathbf{p w}) \forall x, y \in \operatorname{dom} f$,

(i) $x(V)>y(V) \Longrightarrow$

$$
\begin{aligned}
& f(x)+f(y) \\
& \quad \geq \min _{u \in \operatorname{supp}^{+}(x-y)}\left[f\left(x-\chi_{u}\right)+f\left(y+\chi_{u}\right) \underset{v \in \operatorname{supp}^{-}(x-y)}{\min }\left\{f\left(x-\chi_{u}+\chi_{v}\right)+f\left(y+\chi_{u}-\chi_{v}\right)\right\}\right],
\end{aligned}
$$

(ii) $x(V)=y(V), x \neq y \Longrightarrow$

$$
f(x)+f(y) \geq \min _{\substack{u \in \operatorname{supp}^{+}(x-y) \\ v \in \operatorname{supp}^{-}(x-y)}}\left\{f\left(x-\chi_{u}+\chi_{v}\right)+f\left(y+\chi_{u}-\chi_{v}\right)\right\} .
$$

For example, (MG-EXC $)$ (i) is obtained from (MB-EXC) for $\tilde{f}$ with $u=v_{0}$.

The objective of this section is to show that these axioms are equivalent to (MG-EXC) and $\left(\mathrm{MG}-\mathrm{EXC}_{\mathrm{W}}\right)$, which look simpler and nicer.

\footnotetext{
${ }^{1}$ Labels $\left(\mathrm{MB}^{* * *}\right)$ are used for axioms of M-convexity of functions on base polyhedra, while (MG-***) for those on g-polymatroids.
} 
Theorem $4.2(M G-P R J) \Longleftrightarrow(M G-E X C) \Longleftrightarrow\left(M G-E X C_{w}\right) \Longleftrightarrow\left(M G-E X C_{p}\right) \Longleftrightarrow$ $\left(M G-E X C_{p w}\right)$.

We can easily see from definitions and Theorem 4.1 that $\left(\mathrm{MG}-\mathrm{EXC}_{\mathrm{pw}}\right) \Longrightarrow\left(\mathrm{MG}-\mathrm{EXC}_{\mathrm{p}}\right)$ $\Longrightarrow(\mathrm{MG}-\mathrm{EXC}) \Longrightarrow\left(\mathrm{MG}-\mathrm{EXC}_{\mathrm{W}}\right)$. Furthermore, it is obvious that $\left(\mathrm{MG}-\mathrm{EXC}_{\mathrm{W}}\right) \Longrightarrow(\mathrm{MG}-$ $\left.\mathrm{EXC}_{\mathrm{pw}}\right)$ (i). Thus, it suffices to show that $\left(\mathrm{MG}^{-E_{\mathrm{X}}} \mathrm{C}_{\mathrm{w}}\right) \Longrightarrow\left(\mathrm{MG}-\mathrm{EXC}_{\mathrm{pw}}\right)$ (ii). For this purpose, we need some lemmas.

Lemma $4.3\left(M G-E X C_{w}\right) \Longrightarrow \forall x, y \in \operatorname{dom} f$ with $x(V)<y(V)$,

$$
f(x)+f(y) \geq \min _{v \in \operatorname{supp}^{-}(x-y)}\left\{f\left(x+\chi_{v}\right)+f\left(y-\chi_{v}\right)\right\} .
$$

Proof. The proof is similar to and simpler than the one for Lemma 4.6 below and omitted.

Lemma $4.4\left(M G-E X C_{w}\right) \Longrightarrow \forall x, y \in \operatorname{dom} f$ with $x(V)=y(V)$ and $\|x-y\|=4$,

$$
f(x)+f(y) \geq \min _{\substack{u \in \operatorname{supp}^{+}(x-y) \\ v \in \operatorname{supp}^{-}(x-y)}}\left\{f\left(x-\chi_{u}+\chi_{v}\right)+f\left(y+\chi_{u}-\chi_{v}\right)\right\} .
$$

Proof. We can put $x=z+\chi_{w_{1}}+\chi_{w_{2}}, y=z+\chi_{w_{3}}+\chi_{w_{4}}$ with $w_{i} \in V(i=1,2,3,4)$ and $z \in \mathbf{Z}^{V}$ defined by $z(v)=\min \{x(v), y(v)\}$ for $v \in V$. In the following, we denote $\alpha_{1}=f\left(z+\chi_{w_{1}}\right), \alpha_{23}=f\left(z+\chi_{w_{2}}+\chi_{w_{3}}\right), \alpha_{134}=f\left(z+\chi_{w_{1}}+\chi_{w_{3}}+\chi_{w_{4}}\right)$, and so on. To the contrary suppose

$$
\alpha_{12}+\alpha_{34}<\min \left\{\alpha_{13}+\alpha_{24}, \alpha_{14}+\alpha_{23}\right\} .
$$

Then, we have $\alpha_{12}+\alpha_{34}=\min \left\{\alpha_{1}+\alpha_{234}, \alpha_{2}+\alpha_{134}\right\}$. In fact, LHS $\geq$ RHS is by (MG$\left.\mathrm{EXC}_{\mathrm{W}}\right)$ and (2), and the reverse inequality is by Lemma 4.3 and (2). Assume w.l.o.g. that $\alpha_{12}+\alpha_{34}=\alpha_{1}+\alpha_{234}$. From (MG-EXC $\left.\mathrm{W}_{\mathrm{W}}\right)$, it holds that

$2\left(\alpha_{12}+\alpha_{34}\right)=\alpha_{234}+\alpha_{12}+\alpha_{34}+\alpha_{1} \geq \min \left\{\alpha_{123}+\alpha_{24}, \alpha_{124}+\alpha_{23}\right\}+\min \left\{\alpha_{3}+\alpha_{14}, \alpha_{4}+\alpha_{13}\right\}$.

Again assume w.l.o.g. that $\min \left\{\alpha_{123}+\alpha_{24}, \alpha_{124}+\alpha_{23}\right\}=\alpha_{123}+\alpha_{24}$. In case that $\min \left\{\alpha_{3}+\right.$ $\left.\alpha_{14}, \alpha_{4}+\alpha_{13}\right\}=\alpha_{3}+\alpha_{14}$, we have a contradiction since

$$
\alpha_{123}+\alpha_{24}+\alpha_{3}+\alpha_{14} \geq \alpha_{13}+\alpha_{24}+\alpha_{23}+\alpha_{14}>2\left(\alpha_{12}+\alpha_{34}\right),
$$

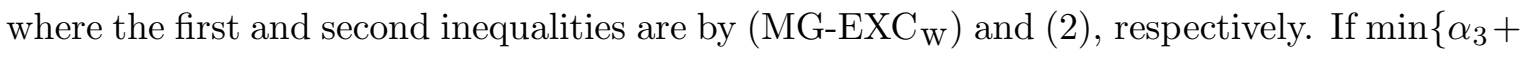
$\left.\alpha_{14}, \alpha_{4}+\alpha_{13}\right\}=\alpha_{4}+\alpha_{13}$, then Lemma 4.3 and (2) yield another contradiction:

$\alpha_{123}+\alpha_{24}+\alpha_{4}+\alpha_{13} \geq \min \left\{\alpha_{12}+\alpha_{34}, \alpha_{13}+\alpha_{24}, \alpha_{14}+\alpha_{23}\right\}+\alpha_{13}+\alpha_{24}>2\left(\alpha_{12}+\alpha_{34}\right)$. 
Lemma $4.5\left(M G-E X C_{w}\right) \Longrightarrow \forall x, y \in \operatorname{dom} f$ with $x(V)=y(V)$ and $x \neq y, \exists u_{1} \in \operatorname{supp}^{+}(x-$ $y), \exists v_{1} \in \operatorname{supp}^{-}(x-y)$ such that $y+\chi_{u_{1}}-\chi_{v_{1}} \in \operatorname{dom} f$.

Proof. By applying (MG-EXC $\mathrm{W}_{\mathrm{W}}$ ) for $x$ and $y$, either (a) or (b) holds, where

(a) $\exists u_{1} \in \operatorname{supp}^{+}(x-y)$ such that $y+\chi_{u_{1}} \in \operatorname{dom} f$,

(b) $\exists u_{1} \in \operatorname{supp}^{+}(x-y), \exists v_{1} \in \operatorname{supp}^{-}(x-y)$ such that $y+\chi_{u_{1}}-\chi_{v_{1}} \in \operatorname{dom} f$.

If (a) holds, then we can apply Lemma 4.3 for $x$ and $y+\chi_{u_{1}}$, which yields that $y+\chi_{u_{1}}-\chi_{v_{1}} \in$ $\operatorname{dom} f$ for some $v_{1} \in \operatorname{supp}^{-}\left(x-\left(y+\chi_{u_{1}}\right)\right) \subseteq \operatorname{supp}^{-}(x-y)$.

In the following, we assume $\left(\mathrm{MG}-\mathrm{EXC}_{\mathrm{W}}\right)$ and show a stronger statement than (MG$\mathrm{EXC}_{\mathrm{pw}}$ ) (ii). The proof is almost the same as the one for [20, Theorem 3.1].

Lemma $4.6\left(M G-E X C_{w}\right) \Longrightarrow \forall x, y \in \operatorname{dom} f$ with $x(V)=y(V), \forall u \in \operatorname{supp}^{+}(x-y)$,

$$
f(x)+f(y) \geq \min _{v \in \operatorname{supp}^{-}(x-y)}\left\{f\left(x-\chi_{u}+\chi_{v}\right)+f\left(y+\chi_{u}-\chi_{v}\right)\right\}
$$

Proof. Set

$$
\begin{aligned}
\mathcal{D}=\{(x, y) \mid x, y & \in \operatorname{dom} f, x(V)=y(V), \exists u_{*} \in \operatorname{supp}^{+}(x-y), \\
& \left.\forall v \in \operatorname{supp}^{-}(x-y): f(x)+f(y)<f\left(x-\chi_{u_{*}}+\chi_{v}\right)+f\left(y+\chi_{u_{*}}-\chi_{v}\right)\right\} .
\end{aligned}
$$

We assume $\mathcal{D} \neq \emptyset$ and derive a contradiction.

Let $(x, y)$ be the element in $\mathcal{D}$ which minimizes the value $\|x-y\|$, and $u_{*} \in \operatorname{supp}^{+}(x-y)$ satisfy the condition for $(x, y)$ to be in $\mathcal{D}$. Using $\varepsilon(>0)$, we set $p \in \mathbf{R}^{V}$ as follows:

$$
p(v)= \begin{cases}f(x)-f\left(x-\chi_{u_{*}}+\chi_{v}\right) & \left(v \in \operatorname{supp}^{-}(x-y), x-\chi_{u_{*}}+\chi_{v} \in \operatorname{dom} f\right), \\ f\left(y+\chi_{u_{*}}-\chi_{v}\right)-f(y)-\varepsilon & \left(v \in \operatorname{supp}^{-}(x-y), x-\chi_{u_{*}}+\chi_{v} \notin \operatorname{dom} f,\right. \\ & \left.y+\chi_{u_{*}}-\chi_{v} \in \operatorname{dom} f\right), \\ & \text { (otherwise). }\end{cases}
$$

Define $f_{p}(x)=f(x)+\sum\{p(w) x(w) \mid w \in V\}\left(\forall x \in \mathbf{Z}^{V}\right)$.

\section{Claim 1}

$$
\begin{array}{ll}
f_{p}\left(x-\chi_{u_{*}}+\chi_{v}\right)=f_{p}(x) & \left(v \in \operatorname{supp}^{-}(x-y), x-\chi_{u_{*}}+\chi_{v} \in \operatorname{dom} f\right) \\
f_{p}\left(y+\chi_{u_{*}}-\chi_{v}\right)>f_{p}(y) & \left(v \in \operatorname{supp}^{-}(x-y)\right)
\end{array}
$$

Suppose that $u_{1} \in \operatorname{supp}^{+}(x-y), v_{1} \in \operatorname{supp}^{-}(x-y)$ satisfy

$$
f_{p}\left(y+\chi_{u_{1}}-\chi_{v_{1}}\right)=\min _{\substack{u \in \operatorname{supp}^{+}(x-y) \\ v \in \operatorname{supp}^{-}(x-y)}} f_{p}\left(y+\chi_{u}-\chi_{v}\right) .
$$

Lemma 4.5 yields that $f_{p}\left(y+\chi_{u_{1}}-\chi_{v_{1}}\right)<+\infty$. Put $y^{\prime}=y+\chi_{u_{1}}-\chi_{v_{1}}$. 
Claim $2\left(x, y^{\prime}\right) \in \mathcal{D}$.

Proof of Claim. We have only to show that

$$
f_{p}(x)+f_{p}\left(y^{\prime}\right)<f_{p}\left(x-\chi_{u_{*}}+\chi_{v}\right)+f_{p}\left(y^{\prime}+\chi_{u_{*}}-\chi_{v}\right)
$$

for each $v \in \operatorname{supp}^{-}\left(x-y^{\prime}\right)$. We can assume that $x-\chi_{u_{*}}+\chi_{v} \in \operatorname{dom} f$, which implies $f_{p}(x)=f_{p}\left(x-\chi_{u_{*}}+\chi_{v}\right)$ by (3) and the fact $v \in \operatorname{supp}^{-}\left(x-y^{\prime}\right) \subseteq \operatorname{supp}^{-}(x-y)$. Furthermore, it holds that

$$
\begin{array}{rlr} 
& f_{p}\left(y^{\prime}+\chi_{u_{*}}-\chi_{v}\right) \\
= & f_{p}\left(y+\chi_{u_{1}}+\chi_{u_{*}}-\chi_{v_{1}}-\chi_{v}\right)+f_{p}(y)-f_{p}(y) \\
\geq & \min \left\{f_{p}\left(y+\chi_{u_{1}}-\chi_{v_{1}}\right)+f_{p}\left(y+\chi_{u_{*}}-\chi_{v}\right),\right. & \\
& \left.\quad f_{p}\left(y+\chi_{u_{1}}-\chi_{v}\right)+f_{p}\left(y+\chi_{u_{*}}-\chi_{v_{1}}\right)\right\}-f_{p}(y) & (\text { by Lemma 4.4) } \\
\geq & f_{p}\left(y^{\prime}\right)+\min \left\{f_{p}\left(y+\chi_{u^{*}}-\chi_{v}\right)-f_{p}(y), f_{p}\left(y+\chi_{u_{*}}-\chi_{v_{1}}\right)-f_{p}(y)\right\} \quad \text { (by (5)) } \\
> & f_{p}\left(y^{\prime}\right) \quad \text { (by (4)), }
\end{array}
$$

which implies the inequality (6).

Hence, we have $\left(x, y^{\prime}\right) \in \mathcal{D}$, and $\left\|x-y^{\prime}\right\|=\|x-y\|-2$, which contradicts the selection of $(x, y)$.

\section{Concluding Remarks}

Remark 5.1 Most properties of M-convex functions on base polyhedra [16, 19, 20, 21] extend to M-convex functions on g-polymatroids, according to its definition. For example,

- an M-convex function on a g-polymatroid is characterized by minimizers,

- M-convexity on g-polymatroids is preserved by addition of a linear function, translation, and negation of the argument,

- an M-convex function on a g-polymatroid can be extended to a convex function,

- convolution and network induction work,

- an intersection theorem, a Fenchel-type duality, and a discrete separation theorem hold.

Remark 5.2 As a corollary of Theorem 4.2, g-polymatroids are characterized by a simultaneous exchange property:

(G-EXC) $\forall x, y \in Q, \forall u \in \operatorname{supp}^{+}(x-y)$, either (i) or (ii) holds, where

(i) $x-\chi_{u} \in Q$ and $y+\chi_{u} \in Q$,

(ii) $x-\chi_{u}+\chi_{v} \in Q$ and $y+\chi_{u}-\chi_{v} \in Q\left(\exists v \in \operatorname{supp}^{-}(x-y)\right)$.

In fact, the axiom (MG-EXC) comes from this characterization. Alternatively, g-polymatroids are characterized by another exchange property: 
(G-EXC $\mathbf{0}) \forall x, y \in Q, \forall u \in \operatorname{supp}^{+}(x-y)$, both (i) and (ii) hold, where

(i) either $x-\chi_{u} \in Q$, or $x-\chi_{u}+\chi_{v} \in Q\left(\exists v \in \operatorname{supp}^{-}(x-y)\right)$,

(ii) either $y+\chi_{u} \in Q$, or $y+\chi_{u}-\chi_{w} \in Q\left(\exists w \in \operatorname{supp}^{-}(x-y)\right)$,

which is a straightforward extension of the one for g-matroids due to Tardos [24]. This axiom, however, is not suitable for a quantitative generalization.

Remark 5.3 Suppose that we are given a function $f: \mathbf{Z}^{V} \rightarrow \mathbf{R} \cup\{+\infty\}$ with (MB-EXC) and a specified subset $W \subseteq V$. Set $\lambda=\min \{x(W) \mid f(x)<+\infty\}, \mu=\max \{x(W) \mid f(x)<+\infty\}$, $\alpha_{k}^{*}=\min \{f(x) \mid x(W)=k\}$, and $M_{k}=\left\{x \in \mathbf{Z}^{V} \mid x(W)=k, f(x)=\alpha_{k}^{*}\right\}$. Then,

$\alpha_{k-1}^{*}+\alpha_{k+1}^{*} \geq 2 \alpha_{k}^{*} \quad(\lambda+1 \leq \forall k \leq \mu-1)$ as in Theorem 3.4, and Theorem 3.3 can be generalized as follows:

Theorem 5.1 Let $x_{k}^{*} \in M_{k}(\lambda \leq k \leq \mu-1)$, and $u \in V-W, v \in W$ be such that $f\left(x_{k}^{*}-\chi_{u}+\chi_{v}\right)=\min \left\{f\left(x_{k}^{*}-\chi_{s}+\chi_{t}\right) \mid s \in V-W, t \in W\right\}$. Then $x_{k}^{*}-\chi_{u}+\chi_{v} \in M_{k+1}$.

Theorems 3.3 and 3.4 are the translation by projection of these results when $|W|=1$. Note that the similar properties of valuated bimatroid in [15] are also the special cases of the above results.

\section{Appendix: Proofs}

\section{A M-convexity of Functions in Examples}

Theorem A.1 Let $f: \mathbf{Z}^{V} \rightarrow \mathbf{R} \cup\{+\infty\}$ be a quasi-separable convex function such that $\operatorname{dom} f$ is a polymatroid. Then, $f$ satisfies $\left(M G-E X C_{w}\right)$.

The proof requires a characterization of polymatroids. See, e.g., Welsh [26].

Lemma A.2 Let $P$ be a nonempty set of nonnegative vectors. Then, $P$ is a polymatroid if and only if $P$ satisfies (P1) and (P2), where

(P1) $y \in P, 0 \leq x \leq y \Longrightarrow x \in P$,

(P2) $\quad x, y \in P, x(V)<y(V) \Longrightarrow \exists v \in \operatorname{supp}^{-}(x-y)$ such that $x+\chi_{v} \in P$.

We also use an exchange axiom $\left(\mathrm{G}-\mathrm{EXC}_{\mathrm{p}}\right)$ for g-polymatroids, which is just a qualitative version of $\left(\mathrm{MG}-\mathrm{EXC}_{\mathrm{p}}\right)$.

$\left(\mathbf{G}-\mathbf{E X C}_{\mathbf{p}}\right) \forall x, y \in Q$,

(i) $x(V)<y(V) \Longrightarrow \exists v \in \operatorname{supp}^{-}(x-y)$ such that $x+\chi_{v} \in Q$ and $y-\chi_{v} \in Q$,

(ii) $x(V) \leq y(V) \Longrightarrow \forall u \in \operatorname{supp}^{+}(x-y), \exists v \in \operatorname{supp}^{-}(x-y) \operatorname{such}$ that $x-\chi_{u}+\chi_{v} \in$ $Q$ and $y+\chi_{u}-\chi_{v} \in Q$,

(iii) $x(V)>y(V) \Longrightarrow \forall u \in \operatorname{supp}^{+}(x-y)$, either $x-\chi_{u} \in Q, y+\chi_{u} \in Q$, or $x-\chi_{u}+\chi_{v} \in Q, y+\chi_{u}-\chi_{v} \in Q$ for some $v \in \operatorname{supp}^{-}(x-y)$. 
Note that any polymatroid is a g-polymatroid and therefore satisfies $\left(\mathrm{G}-\mathrm{EXC}_{\mathrm{p}}\right)$.

Lemma A.3 Suppose $f: \mathbf{Z}^{V} \rightarrow \mathbf{R} \cup\{+\infty\}$ is a quasi-separable convex function such that $\operatorname{dom} f$ is a polymatroid. Let $x, y \in \operatorname{dom} f$.

(a) $x(V)>y(V) \Longrightarrow \exists u \in \operatorname{supp}^{+}(x-y)$ such that $f(x)+f(y) \geq f\left(x-\chi_{u}\right)+f\left(y+\chi_{u}\right)$,

(b) $x(V)=y(V) \Longrightarrow \forall u \in \operatorname{supp}^{+}(x-y), \exists v \in \operatorname{supp}^{-}(x-y)$ such that

$$
f(x)+f(y) \geq f\left(x-\chi_{u}+\chi_{v}\right)+f\left(y+\chi_{u}-\chi_{v}\right) .
$$

Proof. [proof of (a)]: By $\left(\mathrm{G}-\mathrm{EXC}_{\mathrm{p}}\right)(\mathrm{i})$, there exists $u \in \operatorname{supp}^{+}(x-y)$ with $x-\chi_{u} \in$ $\operatorname{dom} f, y+\chi_{u} \in \operatorname{dom} f$. Hence, we have

$$
f(x)-f\left(x-\chi_{u}\right)=\Phi_{u}(x(V)-1, x(u)-1) \geq \Phi_{u}(y(V), y(u))=f\left(y+\chi_{u}\right)-f(y) .
$$

[proof of (b)]: (G-EXCp) (ii) assures the existence of $v \in \operatorname{supp}^{-}(x-y)$ with $x-\chi_{u}+\chi_{v} \in$ $\operatorname{dom} f, y+\chi_{u}-\chi_{v} \in \operatorname{dom} f$. Then, it follows from (P1) that $x-\chi_{u} \in \operatorname{dom} f, y-\chi_{v} \in \operatorname{dom} f$. Thus,

$$
\begin{aligned}
f\left(x-\chi_{u}+\chi_{v}\right)-f(x) & =-\Phi_{u}(x(V)-1, x(u)-1)+\Phi_{v}(x(V)-1, x(v)) \\
& \leq-\Phi_{u}(y(V)-1, y(u))+\Phi_{v}(y(V)-1, y(v)-1) \\
& =f(y)-f\left(y+\chi_{u}-\chi_{v}\right) .
\end{aligned}
$$

This lemma implies (MG-EXC ${ }_{\mathrm{W}}$ ) for quasi-separable convex functions.

Theorem A.4 The function $f_{\mathrm{mcf}}$ in Example 2.2 satisfies (MG-EXC), provided $f_{\mathrm{mcf}}$ does not take the value $-\infty$.

Proof. Let $x, y \in Q, u \in \operatorname{supp}^{+}(x-y)$, and $\varphi_{x}$ and $\varphi_{y}$ be feasible flows with $\partial \varphi_{x}=x$, $\partial \varphi_{y}=y, \Gamma\left(\varphi_{x}\right)=f_{\mathrm{mcf}}(x), \Gamma\left(\varphi_{y}\right)=f_{\mathrm{mcf}}(y)$. Then, we can find $\pi: A \rightarrow\{0, \pm 1\}$ such that

$$
\begin{aligned}
& \operatorname{supp}^{+}(\pi) \subseteq \operatorname{supp}^{+}\left(\varphi_{x}-\varphi_{y}\right), \operatorname{supp}^{-}(\pi) \subseteq \operatorname{supp}^{-}\left(\varphi_{x}-\varphi_{y}\right), \\
& \partial \pi(w)=0 \quad\left(w \in V-\left(V^{+} \cup V^{-}\right)\right), \\
& (\partial \pi)^{-}=\chi_{u}, \text { or }(\partial \pi)^{-}=\chi_{u}-\chi_{v} \text { for some } v \in \operatorname{supp}^{-}(x-y) .
\end{aligned}
$$

Hence $\varphi_{x}-\pi$ and $\varphi_{y}+\pi$ are feasible, and we have either (i) or (ii), where

(i) $\left(\partial \varphi_{x}-\pi\right)^{-}=x-\chi_{u}$ and $\left(\partial \varphi_{y}+\pi\right)^{-}=y+\chi_{u}$,

(ii) $\left(\partial \varphi_{x}-\pi\right)^{-}=x-\chi_{u}+\chi_{v}$ and $\left(\partial \varphi_{y}+\pi\right)^{-}=y+\chi_{u}-\chi_{v}$.

Since

$$
\begin{array}{ll}
f_{a}\left(\varphi_{x}(a)-1\right)+f_{a}\left(\varphi_{y}(a)+1\right) \leq f_{a}\left(\varphi_{x}(a)\right)+f_{a}\left(\varphi_{y}(a)\right) & \text { if } \varphi_{x}(a)>\varphi_{y}(a), \\
f_{a}\left(\varphi_{x}(a)+1\right)+f_{a}\left(\varphi_{y}(a)-1\right) \leq f_{a}\left(\varphi_{x}(a)\right)+f_{a}\left(\varphi_{y}(a)\right) & \text { if } \varphi_{x}(a)<\varphi_{y}(a),
\end{array}
$$


we have

$$
\begin{aligned}
& f_{\mathrm{mcf}}\left(\left(\partial \varphi_{x}-\pi\right)^{-}\right)+f_{\mathrm{mcf}}\left(\left(\partial \varphi_{y}+\pi\right)^{-}\right) \leq \Gamma\left(\varphi_{x}-\pi\right)+\Gamma\left(\varphi_{y}+\pi\right) \\
= & \sum_{a: \pi(a)=1}\left[f_{a}\left(\varphi_{x}(a)-1\right)+f_{a}\left(\varphi_{y}(a)+1\right)\right]+\sum_{a: \pi(a)=-1}\left[f_{a}\left(\varphi_{x}(a)+1\right)+f_{a}\left(\varphi_{y}(a)-1\right)\right] \\
& +\sum_{a: \pi(a)=0}\left[f_{a}\left(\varphi_{x}(a)\right)+f_{a}\left(\varphi_{y}(a)\right)\right] \\
\leq & \Gamma\left(\varphi_{x}\right)+\Gamma\left(\varphi_{y}\right)=f_{\mathrm{mcf}}(x)+f_{\mathrm{mcf}}(y) .
\end{aligned}
$$

Theorem A.5 The function $f_{\text {dis }}$ in Example 2.3 satisfies $(M G-E X C)$.

To prove this, we first show a property on the distance-sum. For $u, v \in V$ with $(u, v) \in E$, set $W(u, v)=\sum\{w(t) \mid t \in V, v \in P(u, t)\}$. For any $u, v \in V$, put $\Delta(u, v)=\sum\left\{l\left(u_{i-1}, u_{i}\right) W\left(u_{i-1}, u_{i}\right) \mid\right.$ $i=1, \cdots, r\}$, where $\left\{u_{0}(=u), u_{1}, \cdots, u_{r}(=v)\right\}$ is the sequence of vertices on the path $P(u, v)$. Note that $\Delta(u, v)$ is not equal to $\Delta(v, u)$.

Lemma A.6 Let $u, v \in V$ and $S$ be a subtree such that $P(u, v) \cap S=\{u\}$. Then,

$$
\operatorname{dis}(S \cup P(u, v))-\operatorname{dis}(S)=-\Delta(u, v) .
$$

Note that the value $\operatorname{dis}(S \cup P(u, v))-\operatorname{dis}(S)$ does not depend on a subtree $S$.

Proof of Theorem A.5 Let $X, Y \in \mathcal{F}$ and $u \in X-Y$. It suffices to show that either (i) or (ii) holds, where

(i) $|X| \geq 3$ and $\operatorname{dis}(S(X))+\operatorname{dis}(S(Y)) \geq \operatorname{dis}(S(X-u))+\operatorname{dis}(S(Y+u))$,

(ii) $\operatorname{dis}(S(X))+\operatorname{dis}(S(Y)) \geq \operatorname{dis}(S(X-u+v))+\operatorname{dis}(S(Y+u-v))(\exists v \in Y-X)$.

For each subtree $S$, we call $w \in S$ a branching vertex of $S$ if there are at least three edges of $S$ incident to $w$.

CASE 1: $S(X)$ and $S(Y)$ contain a common edge.

Let $c$ be the nearest vertex to $u$ in the intersection of $S(X)$ and $S(Y)$. If $|X| \geq 3$, let $b_{X}$ be the nearest branching vertex of $S(X)$ to $u$, and if $|X|=2$ then let $b_{X}$ be the unique element in $X-u$.

CASE 1.1: $b_{X} \in P(c, u)$.

We claim that $|X| \geq 3$. To the contrary suppose $|X|=2$. Then $S(X)=P\left(u, b_{X}\right)$ from the definition of $b_{X}$. But it means that $S(X)$ and $S(Y)$ share no edge, a contradiction. It holds that $S(X-u)=S(X)-P\left(b_{X}, u\right), S(Y+u)=S(Y) \cup P(c, u)$. From Lemma A.6, we have

$$
\begin{aligned}
\operatorname{dis}(S(X-u))+\operatorname{dis}(S(Y+u)) & =\left\{\operatorname{dis}(S(X))+\Delta\left(b_{X}, u\right)\right\}+\{\operatorname{dis}(S(Y))-\Delta(c, u)\} \\
& =\operatorname{dis}(S(X))+\operatorname{dis}(S(Y))-\Delta\left(c, b_{X}\right) \\
& \leq \operatorname{dis}(S(X))+\operatorname{dis}(S(Y)),
\end{aligned}
$$


and the condition (i) is fulfilled.

CASe 1.2: $b_{X} \notin P(c, u)$.

There necessarily exists a leaf $v$ of $S(Y)$ with $c \in P\left(b_{X}, v\right)$. We also have $P(c, v) \cap S(X)=$ $\{c\}$. If there exists a branching vertex of $S(Y)$ on the path $P(c, v)$, let $b_{Y}$ be the nearest one to $v$, and otherwise $b_{Y}=c$. Since $S(X-u+v)=(S(X)-P(c, u)) \cup P(c, v)$, and $S(Y+u-v)=\left(S(Y)-P\left(b_{Y}, v\right)\right) \cup P(c, u)$, Lemma A.6 implies the next inequality:

$$
\begin{aligned}
& \operatorname{dis}(S(X-u+v))+\operatorname{dis}(S(Y+u-v)) \\
= & \{\operatorname{dis}(S(X))+\Delta(c, u)-\Delta(c, v)\}+\left\{\operatorname{dis}(S(Y))+\Delta\left(b_{Y}, v\right)-\Delta(c, u)\right\} \\
= & \operatorname{dis}(S(X))+\operatorname{dis}(S(Y))-\Delta\left(c, b_{Y}\right) \\
\leq & \operatorname{dis}(S(X))+\operatorname{dis}(S(Y)) .
\end{aligned}
$$

Hence $v$ satisfies the condition (ii).

CASE 2: $S(X)$ and $S(Y)$ contain no common edge.

Let $c_{X}$ be the nearest vertex in $S(X)$ to $S(Y)$, and $c_{Y}$ the nearest vertex in $S(Y)$ to $S(X)$. Note that $P\left(c_{X}, c_{Y}\right) \cap S(X)=\left\{c_{X}\right\}$ and $P\left(c_{X}, c_{Y}\right) \cap S(Y)=\left\{c_{Y}\right\}$. If there exists a branching vertex of $S(X)$ on the path $P\left(u, c_{X}\right)$, let $b_{X}$ be the nearest one to $u$, and otherwise $b_{X}=c_{X}$. Let $v$ be any element of $Y$. If there exists a branching vertex on the path $P\left(v, c_{Y}\right)$ then let $b_{Y}$ be the nearest one to $v$, and otherwise set $b_{Y}=c_{Y}$. Since

$$
\begin{aligned}
& S(X-u+v)=\left(S(X)-P\left(b_{X}, u\right)\right) \cup\left(P\left(c_{X}, c_{Y}\right) \cup P\left(c_{Y}, v\right)\right), \\
& S(Y+u-v)=\left(S(Y)-P\left(b_{Y}, v\right)\right) \cup\left(P\left(c_{Y}, c_{X}\right) \cup P\left(c_{X}, u\right)\right),
\end{aligned}
$$

we have the following by Lemma A.6:

$$
\begin{aligned}
& \operatorname{dis}(S(X-u+v))+\operatorname{dis}(S(Y+u-v)) \\
= & \left\{\operatorname{dis}(S(X))+\Delta\left(b_{X}, u\right)-\Delta\left(c_{X}, c_{Y}\right)-\Delta\left(c_{Y}, v\right)\right\} \\
& \quad+\left\{\operatorname{dis}(S(Y))+\Delta\left(b_{Y}, v\right)-\Delta\left(c_{Y}, c_{X}\right)-\Delta\left(c_{X}, u\right)\right\} \\
= & \operatorname{dis}(S(X))+\operatorname{dis}(S(Y))-\Delta\left(c_{X}, c_{Y}\right)-\Delta\left(c_{Y}, c_{X}\right)-\Delta\left(c_{X}, b_{X}\right)-\Delta\left(c_{Y}, b_{Y}\right) \\
\leq & \operatorname{dis}(S(X))+\operatorname{dis}(S(Y)) .
\end{aligned}
$$

This inequality yields that $v$ satisfies the condition (ii).

Theorem A.7 The function $f_{\text {mat }}$ in Example 2.4 satisfies (MG-EXC).

Proof. We define an $m \times(m+n)$ matrix $\widetilde{A}$ as the one consisting of columns in $A$ and unit column vectors $\left\{\chi_{w} \mid w \in R\right\}$. For $J \subseteq C$ and $I \subseteq R$, we denote by $\widetilde{A}[J \cup I]$ a submatrix of $\widetilde{A}$ which contains columns in $A$ corresponding to $J$ and vectors $\left\{\chi_{w} \mid w \in I\right\}$. Then, we have the relations

$$
\operatorname{det} A[I, J]=\operatorname{det} \widetilde{A}[J \cup(R-I)] \quad(I \subseteq R, J \subseteq C,|I|=|J|),
$$


and

$$
f_{\text {mat }}\left(\chi_{J}\right)=-\omega(J) \quad(J \in \mathcal{J}),
$$

where $\omega(J)=\max \left\{\operatorname{deg}_{t} \operatorname{det} \widetilde{A}[J \cup I]|I \subseteq R| I,|+| J \mid=m\right\}$.

Let $J, H \in \mathcal{J}$ and $u \in J-H$. For the M-convexity of $f_{\text {mat }}$, we have only to show that either (i) or (ii) holds, where

(i) $J-u, H+u \in \mathcal{J}$ and $\omega(J)+\omega(H) \leq \omega(J-u)+\omega(H+u)$,

(ii) $\exists v \in H-J$ such that $J-u+v, H+u-v \in \mathcal{J}$ and $\omega(J)+\omega(H) \leq$ $\omega(J-u+v)+\omega(H+u-v)$.

Denote by $I_{J}, I_{H}$ the subsets of $R$ such that

$$
\omega(J)=\operatorname{deg}_{t} \operatorname{det} \widetilde{A}\left[J \cup I_{J}\right], \quad \omega(H)=\operatorname{deg}_{t} \operatorname{det} \widetilde{A}\left[H \cup I_{H}\right] .
$$

Note that both $\widetilde{A}\left[J \cup I_{J}\right]$ and $\widetilde{A}\left[H \cup I_{H}\right]$ are $m \times m$ nonsingular matrices. Since $u \in\left(J \cup I_{J}\right)-$ $\left(H \cup I_{H}\right)$, the Grassmann-Plücker identity guarantees the existence of $v \in\left(H \cup I_{H}\right)-\left(J \cup I_{J}\right)$ such that $\widetilde{A}\left[\left(J \cup I_{J}\right)-u+v\right]$ and $\widetilde{A}\left[\left(H \cup I_{H}\right)+u-v\right]$ are nonsingular and that the following inequality is satisfied (see $[4,5]$ for more detail):

$\operatorname{deg}_{t} \operatorname{det} \widetilde{A}\left[J \cup I_{J}\right]+\operatorname{deg}_{t} \operatorname{det} \widetilde{A}\left[H \cup I_{H}\right] \leq \operatorname{deg}_{t} \operatorname{det} \widetilde{A}\left[\left(J \cup I_{J}\right)-u+v\right]+\operatorname{deg}_{t} \operatorname{det} \widetilde{A}\left[\left(H \cup I_{H}\right)+u-v\right]$.

If $v \in H$ then we have the condition (ii) by (7), (8), and

$\operatorname{deg}_{t} \operatorname{det} \widetilde{A}\left[\left(J \cup I_{J}\right)-u+v\right] \leq \omega(J-u+v), \quad \operatorname{deg}_{t} \operatorname{det} \widetilde{A}\left[\left(H \cup I_{H}\right)+u-v\right] \leq \omega(H+u-v)$,

and if $v \in I_{H}$ then the condition (i) holds by (7), (8), and the following inequalities:

$\operatorname{deg}_{t} \operatorname{det} \widetilde{A}\left[\left(J \cup I_{J}\right)-u+v\right] \leq \omega(J-u), \quad \operatorname{deg}_{t} \operatorname{det} \widetilde{A}\left[\left(H \cup I_{H}\right)+u-v\right] \leq \omega(H+u)$.

Hence, $f_{\text {mat }}$ satisfies (MG-EXC).

\section{B The Proof of Lemma 3.3}

Assuming (MG-EXC $\mathrm{W}_{\mathrm{W}}$ ), we show that for any $x, y \in \operatorname{dom} f$ with $x(V)<y(V)$,

$$
f(x)+f(y) \geq \min _{v \in \operatorname{supp}^{-}(x-y)}\left\{f\left(x+\chi_{v}\right)+f\left(y-\chi_{v}\right)\right\} .
$$

Set

$\mathcal{D}=\left\{(x, y) \mid x, y \in \operatorname{dom} f, x(V)<y(V), \forall v \in \operatorname{supp}^{-}(x-y): f(x)+f(y)<f\left(x+\chi_{v}\right)+f\left(y-\chi_{v}\right)\right\}$.

We assume $\mathcal{D} \neq \emptyset$ and derive a contradiction. 
Let $(x, y)$ be the element in $\mathcal{D}$ which minimizes the value $\|x-y\|$. Using $\varepsilon(>0)$, we set $p \in \mathbf{R}^{V}$ as follows:

$$
p(v)= \begin{cases}f(x)-f\left(x+\chi_{v}\right) & \left(v \in \operatorname{supp}^{-}(x-y), x+\chi_{v} \in \operatorname{dom} f\right), \\ f\left(y-\chi_{v}\right)-f(y)-\varepsilon & \left(v \in \operatorname{supp}^{-}(x-y), x+\chi_{v} \notin \operatorname{dom} f, y-\chi_{v} \in \operatorname{dom} f\right), \\ 0 & \text { (otherwise). }\end{cases}
$$

Define $f_{p}(x)=f(x)+\sum\{p(w) x(w) \mid w \in V\}\left(\forall x \in \mathbf{Z}^{V}\right)$.

\section{Claim 1}

$$
\begin{array}{ll}
f_{p}\left(x+\chi_{v}\right)=f_{p}(x) & \left(v \in \operatorname{supp}^{-}(x-y), x+\chi_{v} \in \operatorname{dom} f\right), \\
f_{p}\left(y-\chi_{v}\right)>f_{p}(y) & \left(v \in \operatorname{supp}^{-}(x-y)\right) .
\end{array}
$$

Claim $2 \exists u_{1} \in \operatorname{supp}^{+}(x-y), \exists v_{1} \in \operatorname{supp}^{-}(x-y)$ such that $y+\chi_{u_{1}}-\chi_{v_{1}} \in \operatorname{dom} f$.

Proof of Claim. Since $y(V)>x(V)$, we can apply $\left(\mathrm{MG}-\mathrm{EXC}_{\mathrm{W}}\right)$ to $y, x$. Combining with the fact $(x, y) \in \mathcal{D}$, there exist $u_{1} \in \operatorname{supp}^{+}(x-y), v_{1} \in \operatorname{supp}^{-}(x-y)$ such that

$$
(+\infty>) f(x)+f(y) \geq f\left(x-\chi_{u_{1}}+\chi_{v_{1}}\right)+f\left(y+\chi_{u_{1}}-\chi_{v_{1}}\right) .
$$

Hence $y+\chi_{u_{1}}-\chi_{v_{1}} \in \operatorname{dom} f$.

Suppose that $u_{1} \in \operatorname{supp}^{+}(x-y), v_{1} \in \operatorname{supp}^{-}(x-y)$ satisfy

$$
f_{p}\left(y+\chi_{u_{1}}-\chi_{v_{1}}\right)=\min _{\substack{u \in \operatorname{supp}^{+}(x-y) \\ v \in \operatorname{supp}^{-}(x-y)}} f_{p}\left(y+\chi_{u}-\chi_{v}\right) .
$$

The previous claim yields that $f_{p}\left(y+\chi_{u_{1}}-\chi_{v_{1}}\right)<+\infty$. Put $y^{\prime}=y+\chi_{u_{1}}-\chi_{v_{1}}$.

Claim $3\left(x, y^{\prime}\right) \in \mathcal{D}$.

Proof of Claim. Since $y^{\prime}(V)=y(V)>x(V)$, we have only to show that

$$
f_{p}(x)+f_{p}\left(y^{\prime}\right)<f_{p}\left(x+\chi_{v}\right)+f_{p}\left(y^{\prime}-\chi_{v}\right)
$$

for each $v \in \operatorname{supp}^{-}\left(x-y^{\prime}\right)$. This inequality stands obviously when $x+\chi_{v} \notin \operatorname{dom} f$. Therefore we assume that $x+\chi_{v} \in \operatorname{dom} f$. We have $f_{p}(x)=f_{p}\left(x+\chi_{v}\right)$ by $(9)$, and

$$
\begin{array}{rlr} 
& f_{p}\left(y^{\prime}-\chi_{v}\right) & \\
= & f_{p}(y)+f_{p}\left(y+\chi_{u_{1}}-\chi_{v_{1}}-\chi_{v}\right)-f_{p}(y) & \\
\geq & \min \left\{f_{p}\left(y+\chi_{u_{1}}-\chi_{v_{1}}\right)+f_{p}\left(y-\chi_{v}\right),\right. & \\
& \left.\quad f_{p}\left(y+\chi_{u_{1}}-\chi_{v}\right)+f_{p}\left(y-\chi_{v_{1}}\right)\right\}-f_{p}(y) & \left(\text { by }\left(\mathrm{MG}-\mathrm{EXC}_{\mathrm{W}}\right)\right) \\
\geq & \min \left\{f_{p}\left(y-\chi_{v}\right)-f_{p}(y), f_{p}\left(y-\chi_{v_{1}}\right)-f_{p}(y)\right\}+f_{p}\left(y+\chi_{u_{1}}-\chi_{v_{1}}\right) & (\text { by }(11)) \\
> & f_{p}\left(y^{\prime}\right) & (\text { by }(10)) .
\end{array}
$$

Thus, the inequality (12) holds.

We have $\left(x, y^{\prime}\right) \in \mathcal{D}$ by Claim 3 , which contradicts the selection of $(x, y)$ since $\left\|x-y^{\prime}\right\|=$ $\|x-y\|-2$. Therefore $\mathcal{D}=\emptyset$. 


\section{References}

[1] Camerini, P. M., M. Conforti, D. Naddef (1989). Some easily solvable nonlinear integer programs. Ricerca Operativa 50 11-25.

[2] Dress, A. W. M., W. Terhalle (1995). Well-layered maps - A class of greedily optimizable set functions. Appl. Math. Lett. 8 77-80.

[3] Dress, A. W. M., W. Terhalle (1995). Well-layered maps and the maximum-degree $k \times k$ subdeterminant of a matrix of rational functions. Appl. Math. Lett. 8 19-23.

[4] Dress, A. W. M., W. Terhalle (1995). Rewarding maps - On greedy optimization of set functions. Adv. Appl. Math. 16 464-483.

[5] Dress, A. W. M., W. Wenzel (1990). Valuated matroid: A new look at the greedy algorithm. Appl. Math. Lett. 3 33-35.

[6] Dress, A. W. M., W. Wenzel (1992). Valuated matroids. Adv. Math. 93 214-250.

[7] Favati, P., F. Tardella (1990). Convexity in nonlinear integer programming. Ricerca Operativa 53 3-44.

[8] Frank, A. (1984). Generalized polymatroids. A. Hajnal et al., eds., Finite and Infinite Sets, North-Holland, Amsterdam, 285-294.

[9] Frank, A., É. Tardos (1988). Generalized polymatroids and submodular flows. Math. Programming 42 489-563.

[10] Fujishige, S. (1984). A note on Frank's generalized polymatroids. Discrete Appl. Math. 7 105-109.

[11] Fujishige, S. (1991). Submodular functions and optimization, North-Holland, Amsterdam.

[12] Korte, B., L. Lovász, R. Schrader (1991). Greedoids, Springer-Verlag, Berlin.

[13] Lovász, L. (1983). Submodular functions and convexity. A. Bachem et al., eds., Mathematical programming - the state of the art, Springer-Verlag, Berlin, 235-257.

[14] Miller, B. L. (1971). On minimizing nonseparable functions defined on the integers with an inventory application. SIAM J. Appl. Math. 21 166-185.

[15] Murota, K. (1995). Finding optimal minors of valuated bimatroids. Appl. Math. Lett. 8 $37-42$. 
[16] Murota, K. (1996). Valuated matroid intersection, I: optimality criteria, II: algorithms. SIAM J. Discrete Math. 9 545-576.

[17] Murota, K. (1997). Matroid valuation on independent sets. J. Combin. Theory Ser. B $6957-78$.

[18] Murota, K. (1996). On exchange axioms for valuated matroids and valuated deltamatroids. Combinatorica 16 591-596.

[19] Murota, K. (1995). Submodular flow problem with a nonseparable cost function, Report No. 95843-OR, Forschungsinstitut für Diskrete Mathematik, Universität Bonn.

[20] Murota, K. (1996). Convexity and Steinitz's exchange property. Adv. Math. $124272-$ 311.

[21] Murota, K. (1996). Discrete convex analysis, RIMS preprint No. 1065, Kyoto University.

[22] Peng, S., A.B. Stephens, Y. Yesha (1993). Algorithms for a core and $k$-tree core of a tree. J. Algorithms 15 143-159.

[23] Shioura, A., T. Uno. A linear time algorithm for finding a $k$-tree-core. J. Algorithms (to appear).

[24] Tardos, É. (1985). Generalized matroids and supermodular colourings. A. Recski, L. Lovász, eds., Matroid Theory, Colloquia Mathematica Societatis János Bolyai 40, NorthHolland, Amsterdam, 359-382.

[25] Tomizawa, N. (1980). Theory of hyperspaces (I) - supermodular functions and generalization of concept of 'bases', Papers of the Technical Group on Circuit and System Theory, Institute of Electronics and Communication Engineers of Japan, CAS80-72. [In Japanese.]

[26] Welsh, D. (1976). Matroid theory, Academic Press, London. 